\title{
Germanica
}

contemporain : jeux intermédiaux, modes de transfert, adaptations

\section{Literatur als „Wiederholungstäterin“ Dürrenmatts Werke zwischen Text und Film}

La littérature comme "récidiviste ». L'ouvre de Dürrenmatt entre texte et film Literature as a persistant offender. Friedrich Dürrenmatt works between text and film

\section{Patricia Linden}

\section{OpenEdition}

\section{Journals}

Édition électronique

URL : http://journals.openedition.org/germanica/2279

DOI : $10.4000 /$ germanica. 2279

ISSN : 2107-0784

Éditeur

Université de Lille

Édition imprimée

Date de publication : 30 décembre 2013

Pagination : 121-135

ISBN : 9782913857322

ISSN : 0984-2632

Référence électronique

Patricia Linden, « Literatur als „Wiederholungstäterin“ Dürrenmatts Werke zwischen Text und Film », Germanica [Online], 53 | 2013, Online erschienen am: 30 Dezember 2016, abgerufen am 06 Oktober 2020. URL : http://journals.openedition.org/germanica/2279 ; DOI : https://doi.org/10.4000/ germanica.2279 


\title{
Literatur als „Wiederholungstäterin“ Dürrenmatts Werke zwischen Text und Film
}

\author{
Patricia LINDEN \\ Universität Antwerpen
}

Die junge Dame: Dürrenmatt hat einen neuen Schluß geschrieben.

Der Autor: Schreibt der immer noch?

Die junge Dame: Nur noch um ${ }^{1}$.

Der Schweizer Autor Friedrich Dürrenmatt schrieb 1958 das Drehbuch für den sehr erfolgreichen Kriminalfilm Es geschah am hellichten Tag. Im gleichen Jahr bearbeitete er den Drehbuchtext für seinen Roman Das Versprechen - Requiem auf den Kriminalroman. Hier kritisiert Dürrenmatt die Trivialisierung des Kriminalromans und - genauer betrachtet - auch die in seinen Augen naive Narrativik, wie sie bei Verfilmungen angewandt wird. Im Roman finden sich zahlreiche Anspielungen auf die Behandlung einer Gattung, die für Dürrenmatt keine gültigen Aussagen mehr machen kann, da sie dem Faktor Zufall, durch die gegebenen äußerlichen Zwänge, nicht den ihm gebührenden Stellenwert einräumen kann: „Ein Geschehen kann schon allein deshalb nicht wie eine Rechnung aufgehen, weil wir nie alle notwendigen Faktoren kennen, sondern nur einige wenige, meistens recht nebensächliche. Auch spielt das Zufällige, Unberechenbare, Inkommensurable

1. - Friedrich Dürrenmatt, Dichterdämmerung, WA= Werkausgabe in 37 Bänden, Zürich: Diogenes 1998. Bd. 9, S. 143 (1956/1980). 
eine zu große Rolle“2. Andererseits nutzt Dürrenmatt die Gattung als Vehikel, um den Leser oder Zuschauer wachzurütteln, da er die Meinung vertritt, dass gerade die scheinbare Leichtigkeit einer Gattung verstören kann. Dürrenmatts Roman, bzw. sein Drehbuch wurden noch fünf Mal verfilmt:

1979 erscheint der zweiteilige italienische Fernsehfilm La Promessa unter der Regie von Alberto Negrin. 1990 verwendet György Fehér den Stoff für die ungarische Produktion Szürkület (Dämmerung), 1994 läuft die niederländisch-amerikanische Koproduktion The Cold Light of Day, von Rudolf van den Berg in den Kinos an. 1997 erscheint dann ein weiteres, diesmal deutsches Remake: Es geschah am hellichten Tag von Bernd Eichinger und Nico Hofmann. Die vorläufig letzte Verfilmung, diesmal als Verfilmung des Romanstoffes und nicht als Remake des Films konzipiert, ist der Film The Pledge aus 2001 von Sean Penn.

Auf diese Art wird also der Kriminalroman, dessen Requiem Dürrenmatt bereits 1958 komponierte, zum filmischen Wiedergänger. Gerade diese Reihenfolge verschiedener Fassungen und Auffassungen, verschiedener „Verbuchungen“3 und Verfilmungen macht den Fall Dürrenmatt interessant für die Adaptionsforschung. Viele Werke von Dürrenmatt waren Anlass zu Adaptionen, sowohl für das Theater als auch für den Film, wobei Der Besuch der alten Dame, der insgesamt sechs Mal verfilmt wurde, besonders erwähnenswert ist.

Der Film von Sean Penn (2001) basiert auf dem Roman Dürrenmatts, während sich die Verfilmung von Nico Hofmann und Bernd Eichinger (1997) eher als Remake des Films von 1958 versteht und sich an das von Dürrenmatt verfasste Drehbuch hält, den Plot gegen Ende aber wesentlich abändert. In der Verfilmung von Rudolf van den Berg wird die Handlung wieder auf andere Weise vom Text losgelöst, während eine gewisse Treue zum Originaldrehbuch bestehen bleibt. Die Handlung wird für die verschiedenen Verfilmungen auch aus dem Schweizer Umfeld jeweils nach Nevada, Deutschland, Südtirol, Ungarn oder in den Ostblock der 70er Jahre verlagert. Die einzige, richtige' Romanverfilmung in dieser Reihe ist die italienische Produktion La Promessa, die alle Elemente aus dem Roman aufgreift und auch die

2. - Friedrich Dürrenmatt, Das Versprechen. Requiem auf den Kriminalroman. Zürich, Diogenes, 1985, S. 13.

3. - „Längst werden nach Filmen und Fernsehspielen Romane geschrieben, Filmdrehbücher werden auf der Bühne adaptiert. Die ,Verbuchung' von Filmen läuft parallel zur Literaturverfilmung (in Kino und Fernsehen). [...] Solch intermediale Vernetzung hat natürlich auch Rückwirkungen auf den Buchmarkt. Selbst von den Gegnern der Literaturverfilmung wird ihre oft auflagensteigernde Servicefunktion als angenehme Begleiterscheinung konzediert.,, Albersmeier, Franz-Josef und Volker Roloff (Hrsg.): Literaturverfilmungen. Frankfurt am Main, Suhrkamp, 1989, S.17 
filmische Darstellung der Rahmenhandlung und deren Gattungskritik mit einbezieht.

Anhand dieses Korpus gehe ich auf folgende Fragen ein: Welche Änderungen am Drehbuch führen zur Romanfassung des Stoffes? Welche Intertextualität verbindet Drehbuch, Roman und die verschiedenen Verfilmungen? Wie kommt es zur textuellen Bindung von Film und Text, bzw. von Übertragung und Transformation? Die nähere Betrachtung der konkreten Text- und Filmgenese möchte eine Anregung für neuere theoretische Ansätze der Film- und Adaptionsanalyse sein.

\section{Drehbuch-Film-Roman}

„Die Transposition eines Stoffes in ein anderes Medium stellt nicht so sehr ein Problem der Phantasie als eines des Denkens dar - zu dem freilich das neue Medium zwingt. Transponieren ist daher oft schwerer als Erfinden 4 ."

1957 wurde Friedrich Dürrenmatt engagiert, um das Drehbuch für einen Film über Sexualverbrechen an Kindern zu schreiben. Während der Arbeit am Drehbuch gab es sehr regelmäßige Kontakte zwischen dem Auftraggeber, Hans Wechsler, und Dürrenmatt. Wechsler ging eigentlich davon aus, dass Dürrenmatt ein Treatment schreibe, während letzterer erst einen Handlungsverlauf mit der Überschrift „Folgende Handlung wäre denkbar“ und dann eine Erzählung, die Dürrenmatt selbst eine „Vorfassung des Romans“5 nennt, schreibt. Die Auftragsarbeit wird genau umschrieben: Dürrenmatt solle einen Film über „Sittlichkeitsverbrechen“6 konzipieren, wobei die Möglichkeiten einer filmischen Übersetzung wie folgt zusammengefasst werden: Der Stoff müsse sich auf „Tatsachen stützen"7 und das Publikum müsse „,informiert, aufgeklärt“ (ebd.) werden. Da Wechsler bereits mehrere aufklärerische Filme produziert hat ${ }^{8}$, hat her eine genaue Vorstellung des Films: dokumentarische und didaktische Elemente sollen mit Spielszenen vermischt werden. Schließlich werden für den Film Ladislao Vajda als Regisseur und Heinz Rühmann als Hauptdarsteller verpflichtet. Rühmann macht zur Bedingung für seine Mitarbeit, dass Hans Jacoby am Drehbuch mitwirke. Jacoby geht hierbei auch auf die Wünsche Rühmanns ein, und man kann wohl kaum verkennen, dass die

4. - Dürrenmatt, WA 16,64.

5. - Das Versprechen, Nachwort.

6. - Oliver Möbert, Intertextualität und Variation im Werk Friedrich Dürrenmatts. Peter Lang Frankfurt a/M, S. 38.

7. - Dürrenmatt 1957a, S. 1.

8. - z.B.Frauennot - Frauenglück (1930), Feind im Blut (1931), Sie fanden eine Heimat (1953) 
Rolle eigens für Rühmann konzipiert wird. Insofern wird Dürrenmatts schriftstellerische Freiheit um ein weiteres eingeengt. Allerdings erklärt Dürrenmatt sich am Ende mit dem Film zufrieden, betont jedoch im Nachwort zum Roman, dass zwar ,der Film meinen Intentionen im wesentlichen entspricht", aber dass „,der Roman einen anderen Weg gegangen ist". In seinem Nachwort weist er auch vorsichtig auf die Folgen der Zusammenarbeit, auf den Film „als eine Kollektivarbeit““ hin. In Interviews äußert sich Dürrenmatt etwas genauer: „Man hätte ruhig frecher und burlesker sein dürfen. Rühmann ist mir zu bürgerlich, zu wenig von der Idee besessen 9 “" In Dürrenmatts Titelvorschlägen, die von den Produzenten strikt abgelehnt wurden, lässt sich unschwer ein gewisser Sarkasmus erkennen: Gott schlief am Vormittag und Schrott geht bummern ${ }^{10}$. Gerade das dem Erwartungshorizont des breiten Publikums entsprechende, politisch korrekte Ende des Films, in dem der Täter bestraft wird und der Kommissär als Held siegt, war für Dürrenmatt eher unbefriedigend. Zwar kann hiermit die Tragik des Geschehens nicht aufgehoben werden, da die Mutter des ermordeten Kindes die Inhaftierung des Täters nicht mehr mitbekommt, aber immerhin siegt schließlich die erhoffte Gerechtigkeit.

Die in der Sekundärliteratur öfters vorgebrachte These, Dürrenmatt habe das Drehbuch größtenteils übernommen, jedoch nur das Ende abgewandelt, lässt sich bei genauerer Betrachtung leicht widerlegen. Das Ende steht keineswegs für sich, sondern bildet eine Einheit mit den anderen Abwandelungen im Buch. Der Kommissär geht an seinem Versprechen zugrunde, der Mörder bleibt unsichtbar, stirbt bei einem Autounfall und kann somit nicht in die Falle gehen. Jegliche Anerkennung bleibt dem Kommissär verwehrt, schlimmer noch, er wird ob seiner unmoralischen Methoden - denen ja eigentlich nur der Wunsch, sein Versprechen einzuhalten, zugrunde liegt - schwer verurteilt. Außerdem verstärkt die Unsichtbarkeit des Mörders den Zweifel an Matthäis psychischer Verfassung. Wir können also sagen, dass Dürrenmatt die für den Film gedachte Erzählung auf intelligenteste Weise dekonstruiert und in der Rahmenerzählung seine Figuren darauf hinweisen lässt, dass Drehbuch und Film eine überwachte Arbeit darstellen, während der Autor eines Romans viel unabhängiger arbeiten kann.

Das häufige Umschreiben des Drehbuchs lässt sich sicherlich auch dadurch erklären, dass Dürrenmatt gleichzeitig den Roman denkt. Insofern sollte man Roman und Film nicht absolut als Ergebnis getrennter Arbeitsstufen betrachten, genauso wenig, wie man von

9. - http://www.welt.de/print/die_welt/hamburg/article112527465/100-JahreGert-Froebe.html . Letzter Zugriff: 8.07.2013

10. - http://cinematographicblog.wordpress.com/2013/02/25/es-geschah-amhellichten-tag/ . Letzter Zugriff: 8.07.2013. 
Verfilmung oder Verbuchung - der Roman als Buch zum Film - reden könnte. In der Sekundärliteratur finden sich verschiedene Spekulationen zur Textgenese von Drehbuch und Roman. Florian Schwarz behauptet in seinem Werk „Der Roman Das Versprechen von Friedrich Dürrenmatt und die Filme Es geschah am hellichten Tag (1958) und The Pledge (2001)“ allerdings: „In der einschlägigen Forschungsliteratur wird der Roman durchweg als Vorlage für die späteren Verfilmungen angesehen [...] Dabei wird der Roman stets als eigenständige literarische Vorlage angesehen ${ }^{11 . " ~ D i e s ~ w i r d ~ b e i ~ g e n a u e r e r ~ B e t r a c h t u n g ~}$ der Forschungsliteratur jedoch gar nicht bestätigt. Textgenese von Drehbuch, Roman und Film werden von den einschlägigen Quellen durchaus korrekt beschrieben. Außerdem formuliert bereits Dürrenmatt selbst die genauen Begebenheiten zum Entstehen des Romans in seinem Nachwort zu demselben. Dürrenmatt dekonstruiert vielmehr die für den Film gedachte Geschichte, entwirft Rahmen- und Binnenhandlung und lässt den Mörder als literarische Figur verschwinden. Ein Ich-Erzähler (ein Autor von Kriminalromanen) führt in die Handlung ein und trifft zufällig auf den Erzähler der Binnenhandlung. Aber auch diese Konstruktion wird von Dürrenmatt regelmäßig zugunsten des Zufalls durchbrochen.

„Ich griff die Fabel aufs Neue auf und dachte sie weiter, jenseits des Pädagogischen. Aus einem bestimmten Fall wurde der Fall des Detektivs, eine Kritik an einer der typischen Gestalten des neunzehnten Jahrhunderts $[\ldots]^{12 *}$

Dürrenmatt möchte sich somit aus den Zwängen der Auftrags- und Kollektivarbeit lösen und eine Gattungskritik formulieren. Insofern geht in den Roman vieles ein, was im Drehbuch keinen Platz fand oder finden durfte. Gerade die überwachte Arbeit am Drehbuch dürfte Anlass gewesen sein, über das Schreiben von Kriminalgeschichten an sich nachzudenken. Der Roman hat eine Rahmenhandlung, in der das Schreiben von Kriminalgeschichten und die Tätigkeit ihrer Hauptperson, des Detektivs (Kommissärs) von Grund auf kritisch dargestellt wird. Außerdem wird der Mörder als sichtbare (und somit auch fassbare) Figur aus der Handlung ausgeschlossen. Er fungiert im Roman als eine Art von Phantom, das ferner nur in Matthäis Gedanken existiert und der Leser sich selbst vorstellen muss.

Dürrenmatt formuliert im Roman seine Zweifel an der Gattung des Kriminalromans und begründet somit auch den Untertitel „Requiem

11. - Florian Schwarz, Der Roman "Das Versprechen" von Friedrich Dürrenmatt und die Filme "Es geschah am hellichten Tag" (1958) und "The Pledge" (2001), Würzburg: Lit Verlag 2007, S. 52

12. - Das Versprechen, Nachwort. 
auf den Kriminalroman.“ Die Rahmenerzählung setzt damit ein, dass deren Ich-Erzähler ,,̈̈ber die Kunst, Kriminalromane zu schreiben, einen Vortrag zu halten" hat ${ }^{13}$. Er kommt bei kaltem, tristen Wetter im provinziellen Saal „des Kaufmännischen Vereins“ an: „Publikum war nur spärlich vorhanden, da gleichzeitig in der Aula des Gymnasiums Emil Staiger über den späten Goethe las." Sein Vortrag ist weder für ihn noch für das Publikum wirklich interessant (,Weder ich noch sonst jemand kam in Stimmung14.") und einige Zuhörer verlassen sogar vorzeitig den Saal. Der „späte Goethe“ scheint beim Publikum jedenfalls beliebter zu sein als der Kriminalroman. Dürrenmatt lässt diesen Vortrag über Goethe übrigens nicht von einer fiktiven Figur abhalten, sondern von dem Schweizer Literaturwissenschaftler Emil Staiger, den Dürrenmatt wegen seiner unkritischen Haltung dem literarischen Kanon gegenüber nicht besonders schätzte ${ }^{15}$. Und in dieser Gegenüberstellung zweier Arten der Literatur gleich am Anfang des Romans, wobei "Goethe" für die ,höhere" stehen soll, und der Kriminalroman für die trivialere, die wenig Beachtung findet, liegt ein weiteres interessantes Thema vor: Dürrenmatt wollte, wie oben bereits erwähnt, im Roman die Sache ,jenseits des Pädagogischen“ behandeln. Gerade eine beliebte, sozusagen leichtere Gattung liefert ihm den Rahmen für seine Dekonstruktionen ${ }^{16}$ :

„Wie besteht der Künstler in einer Welt der Bildung, der Alphabeten? Eine Frage, die mich bedrückt, auf die ich noch keine Antwort weiß. Vielleicht am besten, indem er Kriminalromane schreibt, Kunst da tut, wo sie niemand vermutet. Die Literatur muss so leicht werden, dass sie auf der Waage der heutigen Literaturkritik nichts mehr wiegt: Nur so wird sie wieder gewichtig. ${ }^{17 ،}$

Nach dem Vortrag begibt er sich in sein womöglich noch trostloseres Hotel, wo er an der Bar mit Dr. H., einem ehemaligen Kommandanten der Kantonspolizei Zürich, ins Gespräch kommt. Die Trostlosigkeit setzt sich auf den nächsten Seiten des Romans unvermindert in Betrachtungen und Landschaftsbeschreibungen fort, bis Kommandant und Schriftsteller zusammen an der Tankstelle des vollkommen heruntergekommenen Kommissär Matthäi angelangt sind und der Erzählstrang der Binnenerzählung seinen Anfang in der gleichen

13. - Dürrenmatt WA Bd. 23, S. 11.

14. - Das Versprechen, S. 5.

15. - Möbert, a.a.O., S. 120.

16. - Zitiert nach Roland Bursch Wir dichten die Geschichte: Adaption und Konstruktion von Historie bei Friedrich Dürrenmatt, Würzburg, Königshausen \& Neumann, 2006. S. 126.

17. - So bezeichnete Dürrenmatt auch die Komödie als „Mausefalle“: „Die Komödie muß die Mausefalle sein, in die man das Publikum lockt“. Gespräche I, S. 107. 
Atmosphäre nimmt. So wie der Zufall die beiden zusammen gebracht hat, wird der Zufall auch zum Thema des Verbrechens und seiner Aufklärung. Der Kommandant, der beim Vortrag des Schriftstellers anwesend war, nicht etwa aus Interesse, sondern weil ,ihn das Wetter an der Rückfahrt hinderte" 18 bemerkte im nächtlichen Gespräch in der Hotelbar bereits "Sie tragen ziemlich ungeschickt vor“. Dies ist eine erste, milde Kritik an den Kriminalschriftsteller, die im weiteren Verlauf immer stärker wird: ,[...] hoffen die Leute eben, daß wenigstens die Polizei die Welt zu ordnen verstehe, wenn ich mir auch keine lausigere Hoffnung vorstellen kann ${ }^{19}$."

Dr. H. klärt den Schriftsteller weiter auf:

„Doch wird leider in all diesen Kriminalgeschichten ein noch ganz anderer Schwindel getrieben. Damit meine ich nicht einmal den Umstand, daß eure Verbrecher ihre Strafe finden. Denn dieses schöne Märchen ist wohl moralisch notwendig. (...) all dies will ich durchgehen lassen, und sei es auch nur aus Geschäftsprinzip, denn jedes Publikum und jeder Steuerzahler hat ein Anrecht auf seine Helden und sein Happy-End, sind wir von der Polizei und ihr von der Schriftstellerei gleicherweise verpflichtet ${ }^{20 \text { “ }}$

Diese Aussagen können m.E. nicht nur als Gattungskritik am Kriminalroman, sondern auch als Kritik an der Verfilmung des Drehbuchs verstanden werden. Die Erwähnung des „Steuerzahler(s)“ und des "Geschäftsprinzip(s)“, die ein Happy-End benötigen, sind eindeutig eine Kritik an der Filmindustrie. Interessanterweise ist es der ehemalige Kommandant, also sozusagen derjenige, der sich wirklich mit Kriminalgeschichten auskennt, der dem Schriftsteller die Leviten liest:

„Ich kann mir sogar vorstellen, was Sie sich nun in Ihrem Schriftstellerhirn ausdenken. Man braucht nur, werden Sie sich listigerweise sagen, Matthäi Recht bekommen und den Mörder fangen zu lassen, und schon ergebe sich der schönste Roman oder Filmstoff ... ich kann geradezu voraussagen, dass diese Variante meiner Geschichte so erhebend ist und positiv, dass sie demnächst einfach erscheinen muss, sei es nun als Roman oder als Film 21 “"

Die Kritik gilt einmal der fast vorprogrammierten Überführung des Täters, der Tatsache, dass der Kommissär als Held aus der Geschichte hervorgeht und dem erhebenden und positiven Grundton, der den Film

18. - Zitiert nach Roland Bursch Wir dichten die Geschichte: Adaption und Konstruktion von Historie bei Friedrich Dürrenmatt, Würzburg, Königshausen \& Neumann, 2006. S. 126.

19. - Das Versprechen, S. 6.

20. - Ebd. S. 12.

21. - Ebd. S. 12. Meine Kursivierung, PL. 
begleitet. Unter erhebend kann man dann unschwer politisch korrekt und moralisch indiskutabel verstehen.

Insgesamt gilt, dass der Roman Das Versprechen längst kein Kriminalroman mehr ist, sondern dessen Dekonstruktion. Rein gattungstechnisch betrachtet handelt es sich eher um einen Detektivroman, da die Handlung nicht länger um das Verbrechen an sich, sondern um die Gedanken und Gefühle des Detektivs kreist. Aber auch diese werden dekonstruiert: Matthäi geht an seinem Berufsethos, an seinem Versprechen, den Mörder $\mathrm{zu}$ finden und $\mathrm{zu}$ bestrafen, zugrunde. Der Roman betont den Untergang des Rechercheurs an seinem Engagement, den Fall zu lösen. Diese Geschichte wird dem eher erfolglosen Schriftsteller von einem Ermittler der Kantonspolizei in der Rahmenerzählung erzählt. Die Rahmenerzählung enthält jedoch nicht nur den Dialog zwischen den beiden, sondern auch wesentliche Angaben zum Plot der Geschichte. So wird auch in der Rahmenerzählung der Faktor Zufall, der für den ganzen Roman von wesentlicher Bedeutung ist, eingehend thematisiert. Gerade das Motiv des Spiels der logischen Wahrscheinlichkeit und des Zufalls, der alle Überlegungen herausfordert, ist in Rahmenhandlung wie in Binnenerzählung enthalten und ermöglicht erst die intertextuelle Dekonstruktion. Rahmen- und Binnenerzählung sind aber nicht so scharf voneinander getrennt, wie man auf den ersten Blick annehmen würde: im Erzählfluss sind sie stark verwoben, und schließlich löst die in der Rahmenerzählung enthaltene Geschichte, die Frau Schrott auf ihrem Sterbebett dem Kommandanten erzählt, den Fall unverhofft, auch wenn inzwischen alles zu spät ist.

\section{Intertextualität}

Kerr: „Welche Fassung veröffentlichst Du?“

Dürrenmatt: „Die neue, die dritte. Eigentlich ist das die vierte, denn die erste habe ich auch schon geändert."

Kerr: „Also kommt die zweite, unsere erste, eigentlich die dritte, gar nicht heraus?“"

Dürrenmatt: „Die kommt dann nach meinem Tod noch heraus, für die Germanisten ${ }^{22}$ ““

Sicherlich hatte der Begriff der Intertextualität in den letzten Jahren eine sehr hohe Inflationsrate erlebt. Allgemein steht er für die Beziehungen die Texte zueinander haben und dafür, wie Texte sich gegenseitig beeinflussen. Allerdings ist die Intertextualität beim Medienwechsel, ob man diesen nun Transformation, Adaption oder gar

22. - Friedrich Dürrenmatt und Charlotte Kerr, Rollenspiele, Zürich, Diogenes, 1986, S. 17. 
Translation nennt, eine sehr nützliche Kategorie für die Filmanalyse. Die Forschung hat sich inzwischen intensiv mit Adaptionen beschäftigt. Auf dem Gebiet der filmischen Adaption von Literatur haben insbesondere die Begriffe Transposition, Transformation und auch Translation starken Eingang gefunden. Generell wird gerne die komparative Analyse der unterschiedlichen Textsysteme und ihrer narrativen Strukturen als wichtigste Forschungsmethode angewandt ${ }^{23}$.

Deskriptive Analysen von Filmadaptionen können zeigen, dass Adaptionen sich meistens nicht auf eine treue ,Translation' des literarischen Werks beschränken und sogar Verfilmungen von sehr bekannten literarischen Werken auch andere Quellen anbohren/nützen. Neben diesen Erweiterungen gibt es natürlich die obligatorischen Kürzungen, die meist der erste Gegenstand der Kritik sind.

Dürrenmatts Versprechen weist eine sehr komplexe Form der Intertextualität auf, zumal der Roman gewissermaßen auf einem Drehbuch basiert, jedoch keineswegs als sogenanntes „Buch zum Film“ betrachtet werden darf. Neben der Intertextualität im Roman selber, die durch Märchenmotive, Anspielungen auf Kriminalromane und vieles mehr entsteht, ist für diesen Beitrag vor allem die Intertextualität zwischen dem Drehbuch, dem Film, dem Roman und allen weiteren Verfilmungen von Bedeutung. Eine weitere Form der Intertextualität entsteht durch die kritischen Anspielungen auf die Gattung des Kriminalromans. Lange Zeit galt die Werktreue zum, Original ' innerhalb eines eher moralisierenden Diskurses als wichtigstes Qualitätsmerkmal der Literaturverfilmung. Diese Werktreue angesichts der narrativen, thematischen und ästhetischen Elemente des literarischen Werks ist auch heute noch ein Anliegen vieler Rezeptoren, Kritiker und Forscher ${ }^{24}$, während auch andere Kategorien innerhalb der Literaturund Medienwissenschaft oft noch einen prinzipiellen Charakter bekommen: Äquivalenz, Hierarchisierung von Quellen- und Zieltext, Funktionalität. Dürrenmatt unterläuft in seinen Texten zu Drehbuch und Roman diese Kategorien immer wieder und scheint sie somit schier überflüssig zu machen. In seinem Standardwerk Intertextualität geht Manfred Pfister auf diese Art von intertextueller Verweisung ein: „Schließlich kann intertextuelles Verweisen durch Sinnkontrast

23. - Z.B. Franz-Josef Albersmeier/Volker Roloff, Literaturverfilmungen, Frankfurt, Suhrkamp, 1989; Joachim Paech, Literatur und Film, Stuttgart, Metzler, 1988; Irmela Schneider, Der verwandelte Text, Tübingen, Niemeyer, 1981; Marie-Laure Ryan, Narrative across media: the languages of storytelling, Lincoln, University of Nebraska Press, 2004.

24. - z.B.: Kamilla Elliot, Rethinking the novel/film debate, Cambridge, Cambridge University Press, 2003; Cattrysse Patrick, "Media Translation. Plea for an interdisciplinary approach", In: Versus. Quaderni di studi semiotici, 85 ("Sulla traduzione intersemiotica"), 2002, 251-270. 
zu einer Auf- oder Abwertung des Wirklichkeitsmodells im Folgetext führen.“. In Das Versprechen werde „das neue Wirklichkeitsmodell (des Folgetextes) auch durch die explizite Zurückweisung des Modells des klassischen Detektivromans sichtbar gemacht [...]"25.

In diesem Fall ist also der Roman der Folgetext zum Filmtext, der Filmtext sozusagen das „Original“ und der Roman die Adaption, die eine „explizite Zurückweisung“ dieses „Originals“ formuliert.

Dürrenmatts konträre Haltung gegenüber dem traditionellen Erzählstil ist genügend bekannt. Seine Kriminalromane wurden oft als Anti-Krimis oder als anti-aufklärerisch bezeichnet ${ }^{26}$. Dürrenmatt baute nämlich in die Plots systematisch Zufallsfaktoren zum Vor- oder Nachteil der handelnden Personen ein, um dem Ablauf unvorhersehbare Wendungen zu geben, eben weil er davon überzeugt war, dass ,der Wirklichkeit mit Logik nur teilweise beizukommen" sei.

\section{Die Filme}

„The death of a text through translation is an age-old trope, but it takes on new meaning with its transposition into cinema." 27

Sicherlich sollte man unterscheiden zwischen den Filmen, die auf Dürrenmatts Drehbuch basieren, denjenigen, die auf dem ersten Film und dem Drehbuch basieren und denen, die sich explizit auf den Roman beziehen. Dies sind allesamt Formen der Adaption, mit sehr unterschiedlichen Ausgangspositionen.

Vom Drehbuch gibt es insgesamt vier Fassungen, wobei das jeweilige Ende immer nur auf ausgesprochenes Drängen des Produzenten hin von Dürrenmatt selbst verfasst wurde, dies aber nach den Wünschen der Produktionsgesellschaft und des Hauptdarstellers. Wie bereits erwähnt, kannte das Drehbuch auch verschiedene Entstehungsstufen. Im Auftrag war festgeschrieben, dass aufklärerische wie didaktische Elemente nicht fehlen durften und von dokumentarischem Wissen abgelöst werden sollten ${ }^{28}$. Dem „Sittlichkeitsverbrechen sollte ein „menschliches Gegengewicht" gegenübergestellt werden: die väterliche Liebe eines (kinderlosen) Detektivs zu einem Mädchen ${ }^{29}$. Nach diesen Vorgaben entsteht allmählich die erste Fassung der Filmerzählung, der noch drei

25. - Ulrich Broich / Manfred Pfister (Hg.), Intertextualität. Formen, Funktionen, anglistische Fallstudien, Tübingen, Narr, 1985.

26. - Z.B. Jochen Schmidt in Gangster, Opfer, Detektive, Göttingen, Ullstein Verlag, 1989, S. $567 \mathrm{ff}$.

27. - Abe Mark Nornes, For an Abusive Subtitling. Subtitles of Motion Pictures. In: Film Quarterly, I 1999 S.19

28. - Möbert, S. 38.

29. - Ebd. S. 38f. 
weitere folgen werden. Für die dokumentarische Komponente sammelt Dürrenmatt Materialien bezüglich psychiatrischer Anstalten, der psychischen Verfassung von Triebtätern und polizeiliche Berichte von konkreten Lustmorden ${ }^{30}$. Bei dieser Suche wird er übrigens von Otto Rigenbach, Direktor einer psychiatrischen Klinik, auf Fritz Langs Film M- Eine Stadt sucht einen Mörder (D, 1931) aufmerksam gemacht, wodurch eine weitere Intertextualitätskomponente entsteht.

In seiner umfangreichen Studie Intertextualität und Variation im Werk Friedrich Dürrenmatts skizziert Oliver Möbert eine detaillierte Werkgenese des Drehbuchs zu Es geschah am hellichten Tag. Die Änderungen haben viel öfter mit gesellschaftlicher Akzeptanz zu tun als mit filmischen oder künstlerischen Elementen. So muss zum Beispiel die Szene, in der Kommissär Matthäi sich im Waisenhaus ein Kind beschaffen möchte, gestrichen werden. Der nächste Vorschlag, dass Matthäi sich eine ehemalige Prostituierte mit Kind ins Haus nimmt, wird vom Produzenten genauso wenig akzeptiert ${ }^{31}$. Schließlich werden auch in der vierten Drehbuchfassung von Hans Jacoby noch weitere Änderungen im Sinne Heinz Rühmanns eingebracht. Insgesamt weist der Film von 1958 keine unterschiedlichen Zeitebenen auf und er enthält auch wenige filmische Narrationstechniken. Die Montage ist eine klassische Erzählmontage, in der Kontinuität von Zeit und Raum und logische Übergänge vorherrschen. Ein wichtiger Punkt in diesem Film ist, dass der Zuschauer die Handlung unmittelbar nachvollziehen kann, und nichts dem Zweifel überlassen wird. Im Gegensatz zu den Filmen, die auf dem Roman basieren, gibt es z.B. keine Überlappung zwischen dem Wahrnehmungshorizont des Zuschauers und dem Matthäis.

Die beiden Filme, die sich explizit als Remake des Originalfilms aus dem Jahr 1958 verstehen (Van den Berg 1994; Eichinger/Hoffmann 1997) übernehmen den Titel des Originals, oder wandeln ihn leicht ab (In the Cold light of Day/Tod im kalten Morgenlicht). Der Van den BergFilm verkündet sich selbst sogar als „Das Remake des RÜHMANN/ FRÖBE Films „Es geschah am hellichten Tag“. Jedenfalls haben beide Filme keinen Bezug zum Roman und basieren rein auf dem Drehbuch Dürrenmatts. Diese Filme haben beide ein „Happy End“, insofern der Mörder in eine Falle gelockt wird und der Kommissär den Fall trotz widriger Umstände klärt. Gerade dieses Ende erschien Dürrenmatt realitätsfern. Es passt auch überhaupt nicht in Dürrenmatts dramatisches Konzept, wie er es später in Die Physiker formulierte: „Eine Geschichte ist dann zu Ende gedacht, wenn sie ihre schlimmstmögliche

30. - Ebd., S. 43-45.

31. - Ebd. S. 33-86. 
Wendung genommen hat.“32 Man könnte die Kritik des Dr. H. aus Das Versprechen fast direkt auf diese Art von Kriminalfilmen beziehen:

„Man braucht nur, werden Sie sich listigerweise sagen, Matthäi Recht bekommen und den Mörder fangen zu lassen, und schon ergebe sich der schönste Roman oder Filmstoff ${ }^{33}$.“

Das Drehbuch überlässt nichts dem Zufall, während dieser für Dürrenmatt gerade eine äußerst wichtige Handlungsinstanz ist. Dr. H. kritisiert nochmals den Autor von Kriminalromanen (oder gar Drehbüchern?):

„Ihr baut eure Handlungen logisch auf; wie bei einem Schachspiel geht es zu, hier der Verbrecher, hier das Opfer, hier der Mitwisser, hier der Nutznießer; es genügt, dass der Detektiv die Regeln kennt und die Partie wiederholt, und schon hat er den Verbrecher gestellt, der Gerechtigkeit zum Sieg verholfen. Diese Fiktion macht mich wütend ${ }^{34}$ ““

Es ist, als wollten die weiteren Filme dieser Kritik gerecht werden: Der zweiteilige italienische Fernsehfilm La Promessa ist eine sozusagen „treue“ Adaption des Romans und die einzige Verfilmung, die auch die Rahmenhandlung mit einbezieht. Handlung, Personen und Erzählsituation sind filmisch umgesetzt und stimmen mit denen des Romans überein. Die einzige Nuancenverschiebung liegt im Status des Schriftstellers: dieser ist im Film ein erfolgreicher Kultautor, der vor einem großen, interessierten Publikum einen längeren Vortrag hält. Der ehemalige Kommandant lauscht dem Vortrag vom Restaurant aus. Er belächelt die Aussagen des Schriftstellers und schüttelt ab und zu den Kopf. Die beiden gehen ansonsten nur im Hotel aneinander vorbei und treffen sich zufälligerweise bei einer Autopanne auf der Landstraße wieder, an der Matthäi seine Tankstelle hat. La Promessa setzt die Narrationstechniken des Romans filmisch um und übernimmt die verschiedenen Erzählebenen wie auch das erlebende (der Kriminalautor) und das erzählende Ich (der Kommandant Dr. H.) des Romans. Auch das Setting, das im Roman ebenfalls thematisiert wird ${ }^{35}$, wird im Film angeglichen: der Drehort ist Bozen.

Eine äußerst bemerkenswerte Adaption des Drehbuchs ist der Film des ungarischen Regisseurs György Fehér, Szürkület. Szürkulet ist ein außergewöhnlicher Film, der zwar auf dem Drehbuch von

32. - Dürrenmatt, 21 Thesen, Punkt 3, Die Physiker, Anhang. WA, Bd.7

33. - Das Versprechen, S. 132f.

34. - Das Versprechen, S. $15 \mathrm{f}$.

35. - Z.B.: "Sein Verstand war überragend, doch durch das allzu solide Gefüge unseres Landes gefühllos geworden." Ebd., S. 14. 
Dürrenmatt basiert, sich aber einer sehr filmischen Narrativik bedient. Die Geschichte wird hauptsächlich in schattenhaften SchwarzweißBildern erzählt, ständig von Dröhnen, Regengeräuschen und unheimlichen Gesängen untermalt. Die Kameraführung ist unendlich langsam: der ganze Film enthält nicht mehr als 50 Shots, die langsam ineinander übergleiten. In jeder Szene wird ein Puzzlestück zum Fall gezeigt. Als Adaption ist diese Produktion besonders interessant, da sie einerseits eine bestimmte Treue zum Drehbuch aufweist, was die Handlung anbelangt, diese andererseits aber völlig neu schreibt. Wenn man die Geschichte nicht bereits kennt, könnte man sie nur schwer aus dem Film heraus verstehen:

„Fehér didn't choose to draw attention to the plot's development at all. What the spectators are invited to examine is not a crime story, as they would be led to believe. The story is simply a frame or, rather, a leitmotif bringing together the various sequences. What Fehér composed is a visual suite, an entrancing trip through the dusky territories of the human soul.“"36

Die Geschichte wird hier als eine Art Leitmotiv dargestellt, das verschiedene Sequenzen zusammenbringen soll. Der Film wird zur visuellen Suite, zu einem bewegenden Trip durch die staubigen Regionen der menschlichen Seele ${ }^{37}$. Dieser Film hebt sich quasi völlig von seiner Vorlage ab und reduziert die literarische Narrativik auf das reine Zusammenspiel von Bild und Ton. Auch Kausalität und Erzählstrang werden in diesem Film dekonstruiert; Erwartungen werden nirgends erfüllt. Die Kamera filmt „,die Gesichter der Schauspieler als seien es Landschaften und die Landschaften als seien es Gesichter ${ }^{38}$ “" Hier finden wir also die Adaption einer Kriminalgeschichte, die diese ohne jede „Action“ umsetzt. Hier sucht die Kamera den Mörder in den Gedanken der Personen und in den dunklen Landschaften, der Text ist zum Bild geworden ${ }^{39}$.

Die vorläufig letzte Verfilmung des Stoffes ist Sean Penns The Pledge, die sich wiederum explizit als Verfilmung des Romans versteht und die Handlung nach Nevada verlegt. Im Mittelpunkt steht die tragische Person des Kommissärs, von Jack Nicholson gespielt, der genau am Tage seiner Pensionierung mit dem Mord an Gritli Moser konfron-

36. - Christi Puiu: http://icelandchronicles.com/2010/10/riff-2010-szurkulet-bygyorgy-feher/ http://thequietus.com/articles/09594-gyorgy-feher-twilight-szurkulethorse-hospital-screening

37. - Meine Übersetzung, PL.

38. - Meine Übersetzung von: ,The eye of the camera silently explores the actors' faces like they were landscapes and landscapes like they were actors." Christi Puiu: http:// icelandchronicles.com/2010/10/riff-2010-szurkulet-by-gyorgy-feher/

39. - Ebd. 
tiert wird, und - obschon er nicht mehr in Dienst ist - sein Versprechen unbedingt halten will. An dem Zwiespalt zwischen diesem Versprechen und der Liebe zu Frau und Kind geht er zu Grunde. Die Verfilmung reduziert die Rahmenhandlung des Romans wesentlich, verzichtet aber nicht gänzlich auf sie. Ansonsten setzt der Film die Akzente sehr ähnlich wie der Roman. Auch die zeitlichen Ebenen sind nicht linear angeordnet und die Narrativik benutzt Vorausdeutungen, Flash-backs, Träume und Brüche.

\section{Fazit}

STIMME GREEN Du hast mich erfunden.

F.D. Gefunden.

STIMME GREEN Da sollte ich doch wissen, wie ich aussehe.

F.D. Mir scheißegal.

STIMME GREEN Die Zuschauer sitzen im Stockdunkeln vor einer schwarzen Leinwand.

$F$. D. So säße ich manchmal auch ganz gern im Kino.

STIMME GREEN Ich muß endlich auf der Leinwand erscheinen.

F.D. Niemand hindert dich daran.

STIMME GREEN Ich weiß ja nicht, wie ich aussehe.

F. D. Ich auch nicht ${ }^{40}$.

Das obige Zitat aus Midas oder die schwarze Leinwand - von dem es übrigens nicht weniger als 14 Fassungen gibt - zeigt eindeutig, dass Dürrenmatt sowohl seinem „Umschreiben“ als auch der Transposition seiner Werke weder gleichgültig noch humorlos gegenüberstand. Hier nimmt er sarkastisch Stellung zur filmischen Umsetzung seiner Figuren und deutet an, dass das filmisch sichtbar machen seiner literarischen Personen und Handlungen ihm ,scheißegal“" ist und er auch gerne mal „im Stockdunkeln vor einer schwarzen Leinwand“ sitzen würde.

Dies ist der letzte Text Dürrenmatts vor seinem Tod im Jahr 1990. Er bezeichnete ihn selbst als einen „Film zum Lesen“41. Es ist eine Art von Drehbuch, das sich nicht verfilmen lässt, obschon die Kriminalgeschichte, die hier erzählt werden soll, denkbar einfach ist. Im Drehbuch diskutiert der Autor, „F.D.“, in ironischen Dialogen mit seinen Rollen, bis die Geschichte an sich nicht mehr von der Metaebene

40. - Dürrenmatt, WA 26, 140f.

41. - Vgl. Ulrich Weber und Anna von Planta: Nachweis zu Publikationsgeschichte sowie zur Textgrundlage, in: Friedrich Dürrenmatt: Minotaurus. Der Auftrag. Midas. Zürich, Diogenes 1998 [=Werkausgabe Bd. 26]. S. 195-196. 
unterschieden werden kann. Dürrenmatt hatte tatsächlich vor, diesen Text auch zu verfilmen, und schrieb eine ,brauchbare ' Drehbuchfassung, die jedoch nie verfilmt wurde. Sonst wäre sicherlich auch dieser Text zum „Wiederholungstäter“ geworden. 
